2004

\title{
Exchange bias and enhancement of the Néel temperature in thin $\mathrm{NiF} 2$ films
}

Hongtao Shi

D. Lederman

K. V.O’Donovan

J.A. Borchers

Follow this and additional works at: https://researchrepository.wvu.edu/faculty_publications

\section{Digital Commons Citation}

Shi, Hongtao; Lederman, D.; O’Donovan, K. V.; and Borchers, J. A., "Exchange bias and enhancement of the Néel temperature in thin NiF 2 films" (2004). Faculty Scholarship. 159.

https://researchrepository.wvu.edu/faculty_publications/159 


\title{
Exchange bias and enhancement of the Néel temperature in thin $\mathrm{NiF}_{2}$ films
}

\author{
Hongtao Shi and D. Lederman \\ Department of Physics, West Virginia University, Morgantown, West Virginia 26506-6315, USA \\ K. V. O’Donovan* \\ Department of Materials Science and Engineering, University of Maryland, College Park, Maryland 20742-2115, USA \\ and National Institute of Standards and Technology, Gaithersburg, Maryland 20899, USA \\ J. A. Borchers \\ National Institute of Standards and Technology, Gaithersburg, Maryland 20899, USA \\ (Received 29 December 2003; revised manuscript received 25 March 2004; published 21 June 2004)
}

\begin{abstract}
Epitaxial thin (110) films of the weak ferromagnet $\mathrm{NiF}_{2}$ were deposited on single-crystal $\mathrm{MgF}_{2}$ (110) substrates via molecular-beam epitaxy. Subsequently polycrystalline Co was grown on the $\mathrm{NiF}_{2}$ film. The antiferromagnetic ordering of the $\mathrm{NiF}_{2}$ was monitored as a function of temperature via neutron diffraction and the exchange bias $H_{E}$ of the Co layers was measured via standard magnetometry measurements. Because in $\mathrm{NiF}_{2}$ the spins lie in the (001) plane, the maximum $H_{E}$ is observed after cooling the sample with a cooling field in the film plane perpendicular to the [001] direction of the $\mathrm{NiF}_{2}$. In $60 \mathrm{~nm}, 49 \mathrm{~nm}$, and $38 \mathrm{~nm}$ thick $\mathrm{NiF}_{2}$ samples, the Néel temperature is $T_{N} \approx 81 \mathrm{~K}$, which is significantly larger than the bulk value of $T_{N}=73.2 \mathrm{~K}$. This enhancement also occurs in films without Co overlayers and thus is not due to a proximity effect. For the $38 \mathrm{~nm}$ sample with a Co overlayer cooled in a $50 \mathrm{kOe}$ field, $H_{E}>0$ and vanishes at a blocking temperature $\left(T_{B}\right)$ which coincides with the $T_{N}$ of the films. When the sample is cooled in $2.0 \mathrm{kOe}, H_{E}<0$, disappearing at $T=55 \mathrm{~K}$, reappearing at $T=65 \mathrm{~K}$, and finally disappearing once again at $T=81 \mathrm{~K}$. For the $12 \mathrm{~nm}$ thick $\mathrm{NiF}_{2}$ sample, $T_{B} \ll T_{N}$. Strain-induced enhancement of ferromagnetic exchange interactions between the nearestneighbor $\mathrm{Ni}^{2+}$ ions along the $c$ axis may be responsible for the $T_{N}$ enhancement. These results also demonstrate that in general, a diminished $T_{B}$ is not necessarily due to a lower $T_{N}$.
\end{abstract}

DOI: 10.1103/PhysRevB.69.214416 PACS number(s): 75.70.Cn, 75.25.+z, 75.30.Gw, 75.50.Ee

\section{INTRODUCTION}

Exchange bias $\left(H_{E}\right)$ refers to the shift of the magnetic hysteresis loop of a ferromagnet $(\mathrm{F})$ away from $H=0$ when the $\mathrm{F}$ is coupled to an antiferromagnet $(\mathrm{AF})$ or a ferrimagnet. It was originally discovered in Co particles whose surface was oxidized to form $\mathrm{CoO} .{ }^{1}$ This effect has been observed in a large number of systems, including ferrimagnetic/ ferromagnetic bilayers. ${ }^{2,3} H_{E}$ vanishes above a temperature conventionally denoted as the blocking temperature $T_{B}$. Bilayer systems in which the $\mathrm{AF}$ is below a critical thickness $t_{C}$, which is system dependent, generally have a much lower $T_{B}$ compared to the Néel ordering temperature $T_{N}$ of the bulk AF. ${ }^{4,5}$ This reduction of $T_{B}$ could be due to the decrease of $T_{N}$ because of finite size or strain effects, ${ }^{6,7}$ but it is often difficult to unambiguously distinguish between $T_{B}$ and $T_{N}$. By performing neutron diffraction on single crystalline $\mathrm{Fe}_{3} \mathrm{O}_{4} / \mathrm{CoO}$ multilayers, it was recently shown that the $T_{N}$ of $\mathrm{CoO}$ layers increases when the thickness of $\mathrm{CoO}$ is less than $5 \mathrm{~nm}$, whereas $T_{B}$ decreases in that thickness regime. ${ }^{8}$ This increase in $T_{N}$ was interpreted to arise from the proximity of the $\mathrm{CoO}$ layers to the ferrimagnetic $\mathrm{Fe}_{3} \mathrm{O}_{4}$ layers, which have a much higher-ordering temperature than $\mathrm{CoO}$ (for bulk $\mathrm{Fe}_{3} \mathrm{O}_{4} T_{C}=858 \mathrm{~K}$, and for bulk $\mathrm{CoO} T_{N}=291 \mathrm{~K}$ ). It was also recently reported that the $\mathrm{FeF}_{2}$ thin film $T_{N}$ can be enhanced to $T_{N} \sim 82 \mathrm{~K}$ (bulk $T_{N}=78.4 \mathrm{~K}$ ) when $\mathrm{FeF}_{2} / \mathrm{ZnF}_{2}$ multilayers are grown on $\mathrm{MgO}$ (100) substrates with $\mathrm{FeF}_{2}$ layer thicknesses between 10 and 15 monolayers. ${ }^{9}$ This enhancement was attributed to the strain in $\mathrm{FeF}_{2}$ due to the small lattice mismatch between these two materials.
In this paper we report on the epitaxial growth and exchange bias of the weak ferromagnet $\mathrm{NiF}_{2}$. We find several interesting new phenomena, including: (1) a significant increase in $T_{N}$ with respect to bulk, possibly due to strain; (2) a significant exchange bias in $\mathrm{NiF}_{2}$ films with Co overlayers; (3) a reentrant exchange bias behavior for intermediate (38 nm) $\mathrm{NiF}_{2}$ thin-film thicknesses; and (4) a significant difference in the $T_{N}$ and $T_{B}$ for thin $\mathrm{NiF}_{2}$ samples $(12 \mathrm{~nm})$. These results are discussed in terms of the known magnetic properties of $\mathrm{NiF}_{2}$.

The properties of $\mathrm{NiF}_{2}$ in bulk form can be summarized as follows. $\mathrm{NiF}_{2}$ shares the rutile crystal structure with $\mathrm{MgF}_{2}$, $\mathrm{FeF}_{2}, \mathrm{MnF}_{2}, \mathrm{CoF}_{2}$, and $\mathrm{ZnF}_{2}$, having lattice constants $a=b$ $=0.4651 \mathrm{~nm}$ and $c=0.3084 \mathrm{~nm}$ at room temperature. ${ }^{10}$ For $\mathrm{NiF}_{2}$ the magnetic Hamiltonian is ${ }^{11}$

$$
\begin{aligned}
\mathcal{H}= & -\sum_{i, j, i \neq j} J_{i j} \vec{S}_{i} \cdot \vec{S}_{j}+A \sum_{i} S_{i z}^{2} \\
& +E\left[\sum_{i}\left(S_{i x^{\prime}}^{2}-S_{i y^{\prime}}^{2}\right)-\sum_{j}\left(S_{j x^{\prime}}^{2}-S_{j y^{\prime}}^{2}\right)\right],
\end{aligned}
$$

where $J_{i j}$ are magnetic exchange interactions between $\mathrm{Ni}^{2+}$ ions, $A$ is a single-ion magnetic anisotropy, $E$ is an additional anisotropy term arising from spin-orbit coupling in the $\mathrm{Ni}^{2+}$ ions, and the $x^{\prime}$ and $y^{\prime}$ axes are at $45^{\circ}$ relative to the crystalline $a$ and $b$ axes. Also in the third term, the sums over spins $i$ and $j$ refer to spins on opposite sublattices. Because the single-ion anisotropy term $A$ is positive, at low tempera- 


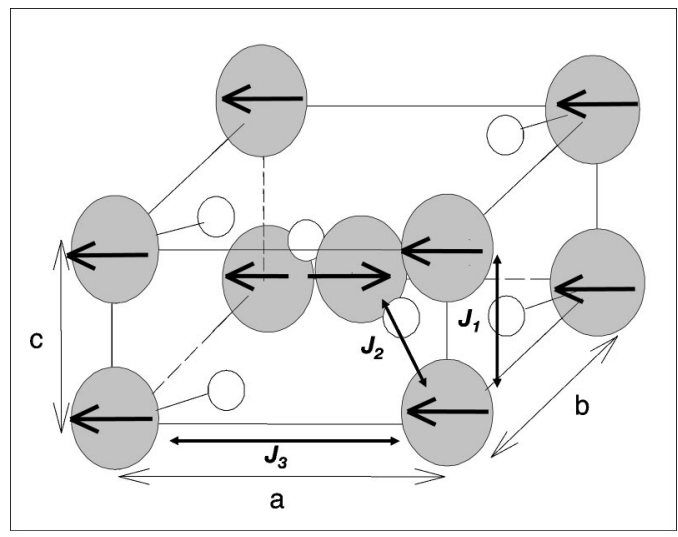

FIG. 1. Crystalline $\mathrm{NiF}_{2}$ structure (small and large circles represent $\mathrm{F}^{-}$and $\mathrm{Ni}^{2+}$ ions, respectively) with one possible magnetic arrangement at low temperatures. Another possible arrangement is with the spins along the $b$ axis. The exchange interactions $J_{1}, J_{2}$, and $J_{3}$ are indicated.

tures the spins lie in the (001) plane, in contrast to $\mathrm{FeF}_{2}$, $\mathrm{MnF}_{2}$, and $\mathrm{CoF}_{2}$, where $A<0$ (resulting in spins along the [001] direction). The competition between the rhombic term $E$ (in the other fluorides $\sim 0$ ), which tends to align the spins perpendicular to each other, and the exchange term, which tends to align them antiparallel to each other, causes a canting of the spins in the $a-b$ plane. ${ }^{12}$ Because $E \ll J z$, with $z$ $=8$ being the number of next-nearest neighbors for each site, the canting angle is small, approximately $0.43^{\circ} .{ }^{12}$ This results in a small spontaneous magnetic moment, and hence $\mathrm{NiF}_{2}$ is a weak ferromagnet. Single-crystal samples in principle can be made into a single magnetic domain by applying a large field along the [100] or [010] direction below $T_{N}{ }^{13}$ The value of $T_{N}=73.2 \mathrm{~K}$ has been verified on bulk single crystals via heat capacity, ${ }^{13}$ magnetization, ${ }^{14}$ and thermal expansion $^{15}$ measurements. The low-temperature magnetic structure and the dominant exchange interactions are shown in Fig. 1.

The exchange constants have been determined from inelastic neutron scattering: ${ }^{12} J_{1}=0.22 \mathrm{~cm}^{-1}$ (ferromagnetic coupling between ions along the $c$ axis, nearest neighbors), $J_{2}=-13.87 \mathrm{~cm}^{-1}$ (antiferromagnetic coupling between corner to body center ions, next-nearest neighbors), $J_{3}=-0.79$ $\mathrm{cm}^{-1}$ (antiferromagnetic coupling between ions along the $a$ or $b$ axis, next-next-nearest neighbors). Note that $J_{2} \gg J_{1}, J_{3}$, and thus dominates the exchange interactions. Spins in (110) planes are almost compensated, except for the small spontaneous canting, since the magnetic moments of the $\mathrm{Ni}^{2+}$ ions on the vertices of the body-centered tetragonal (bct) lattice tend to point opposite to those at the center of the unit cell.

\section{EXPERIMENTAL DETAILS}

\section{A. Growth}

All samples were grown on commercially grown and polished (110) $\mathrm{MgF}_{2}$ single-crystal substrates by molecularbeam epitaxy (MBE) at a growth rate of $\sim 0.02 \mathrm{~nm} / \mathrm{s}$, monitored by quartz-crystal monitors. The substrate was rinsed in methanol for $10 \mathrm{~min}$ prior to transfer to the MBE chamber with a base pressure of $1.0 \times 10^{-9}$ mbar. Before the deposition, the substrate was heated to $297^{\circ} \mathrm{C}$ for $30 \mathrm{~min}$. $\mathrm{NiF}_{2}$ was then deposited onto the substrate by electron-beam evaporation of compressed $\mathrm{NiF}_{2}$ pellets. The growth pressure during evaporation was $\sim 5.0 \times 10^{-8}$ mbar. After the growth of the $\mathrm{NiF}_{2}$ layer, a polycrystalline Co film, with a nominal thickness of $18 \mathrm{~nm}$, was deposited at $125^{\circ} \mathrm{C}$. The actual thicknesses were measured after growth via $\mathrm{x}$-ray reflectivity, as discussed below. In order to prevent oxidation, all samples were capped with $5 \mathrm{~nm} \mathrm{MgF}_{2}$ deposited at room temperature.

\section{B. Structural characterization}

The surface crystal structure was analyzed in situ via reflection high-energy electron diffraction (RHEED). The crystallography and interface structure was analyzed ex situ from $\mathrm{x}$-ray diffraction and reflectivity data, obtained from a rotating anode source using $\mathrm{Cu} K \alpha$ radiation. In-plane lattice parameters were determined from Bragg reflections with a component of the x-ray momentum-transfer vector $q$ pointing in the plane of the sample. ${ }^{16,17}$ Reflectivity data were fit to a recursive optical model to determine the thickness of each layer, as well as the interface roughness between adjacent layers. $^{18}$

\section{Magnetization measurements}

The exchange bias was measured in a superconducting quantum interference device (SQUID) magnetometer after field cooling the sample from $T=100 \mathrm{~K}$ to $T=5 \mathrm{~K}$. Both the cooling field $H_{C F}$ and the measuring field $H$ were applied parallel to the $\mathrm{NiF}_{2}$ [110] direction, that is, in the plane of the sample and perpendicular to the $c$ axis. No exchange bias was observed if $H_{C F}$ was applied parallel to the $c$ axis. This may occur because the moments in the $\mathrm{F}$ and AF layers are perpendicular to each other during cooling in this situation, so that the net interface interaction between the antiferromagnet and the ferromagnet is $J_{I} \vec{S}_{F} \cdot \vec{S}_{A F}=0$, where $J_{I}$ is an effective interface exchange interaction and $\vec{S}_{F}$ and $\vec{S}_{A F}$ are the spins in the $\mathrm{F}$ and $\mathrm{AF}$ layers, respectively. ${ }^{19}$

Additional measurements were carried out using a vibrating sample magnetometer (VSM) which allowed us to cool the sample in a magnetic field and then rotate the sample at low temperatures in order to measure $M \perp H_{C F}$.

\section{Magnetic neutron diffraction}

Neutron-diffraction measurements were carried out at the NIST Center for Neutron Research. The BT-2 and BT-9 triple-axis spectrometers were used with a neutron wavelength of $0.235 \mathrm{~nm}$. A closed cycle refrigerator was used to cool the sample to $12 \mathrm{~K}$. The $(001) \mathrm{NiF}_{2}$ magnetic Bragg reflection, which is sensitive to the ordering of spins in the (001) planes, was monitored as a function of temperature in order to determine $T_{N}$. Note that the existence of this reflection also proves that the magnetic order in the film is that of $\mathrm{NiF}_{2}$. This peak is normally absent for the other antiferromagnetic rutile fluorides $\left(\mathrm{FeF}_{2}, \mathrm{MnF}_{2}\right.$, and $\left.\mathrm{CoF}_{2}\right)$ due to the 


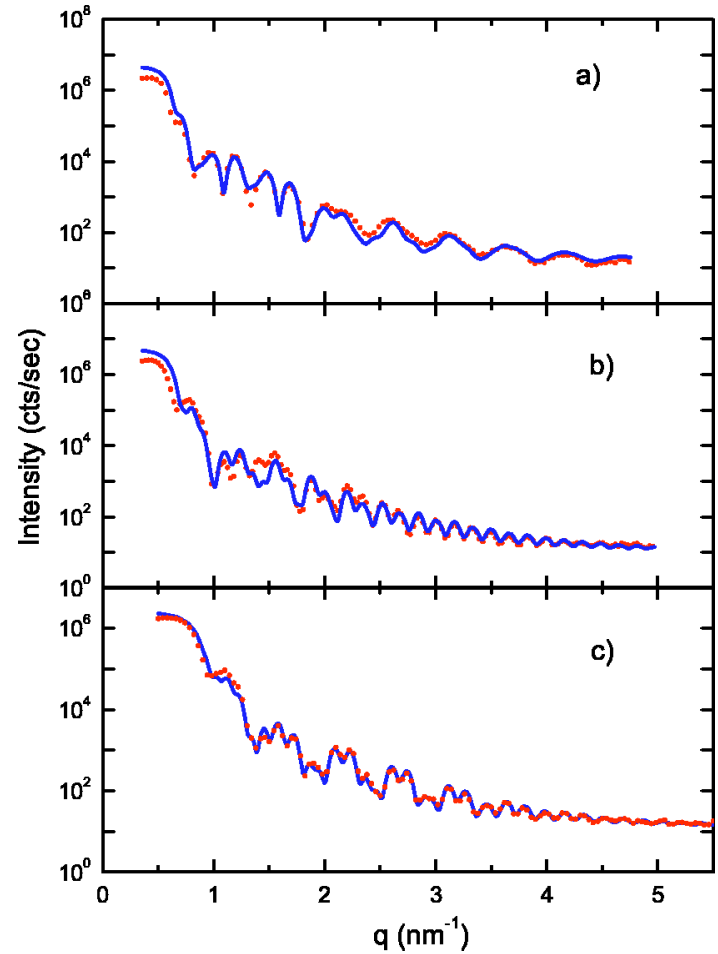

FIG. 2. Specular x-ray reflectivity intensity as a function of the X-ray wave vector $\vec{q}$ for $\mathrm{NiF}_{2} / \mathrm{Co}$ bilayers with $\mathrm{NiF}_{2}$ thicknesses of (a) $12 \mathrm{~nm}$, (b) $38 \mathrm{~nm}$, and (c) $49 \mathrm{~nm}$. The dots are the acquired data and the solid lines are fits to a fully optical reflectivity model. The interface roughness parameters resulting from the fit are shown in Table I. For (a) and (b) the fitted intensity below the critical edge is larger than the measured data because at those angles that size of the sample was smaller than the x-ray beam footprint.

neutron magnetic scattering selection rules for their localized spins pointing along the [001] direction. ${ }^{20}$

\section{RESULTS AND DISCUSSION}

\section{A. Structure}

Given that epitaxial growth of $\mathrm{NiF}_{2}$ films has not, to the best of our knowledge, been reported previously, we present a detailed description of the structure of our films.

Figure 2 shows $x$-ray reflectivity scans for three $\mathrm{NiF}_{2} / \mathrm{Co}$ bilayers. The fit parameters are summarized in Table I. The fits show that the roughness at the $\mathrm{NiF}_{2} / \mathrm{Co}$ interface is $\approx 0.3 \mathrm{~nm}$, which is significantly smaller than the roughness observed in single-crystal $\mathrm{FeF}_{2} / \mathrm{Co}$ bilayers $(\sigma \approx 0.8 \mathrm{~nm}) .^{21}$

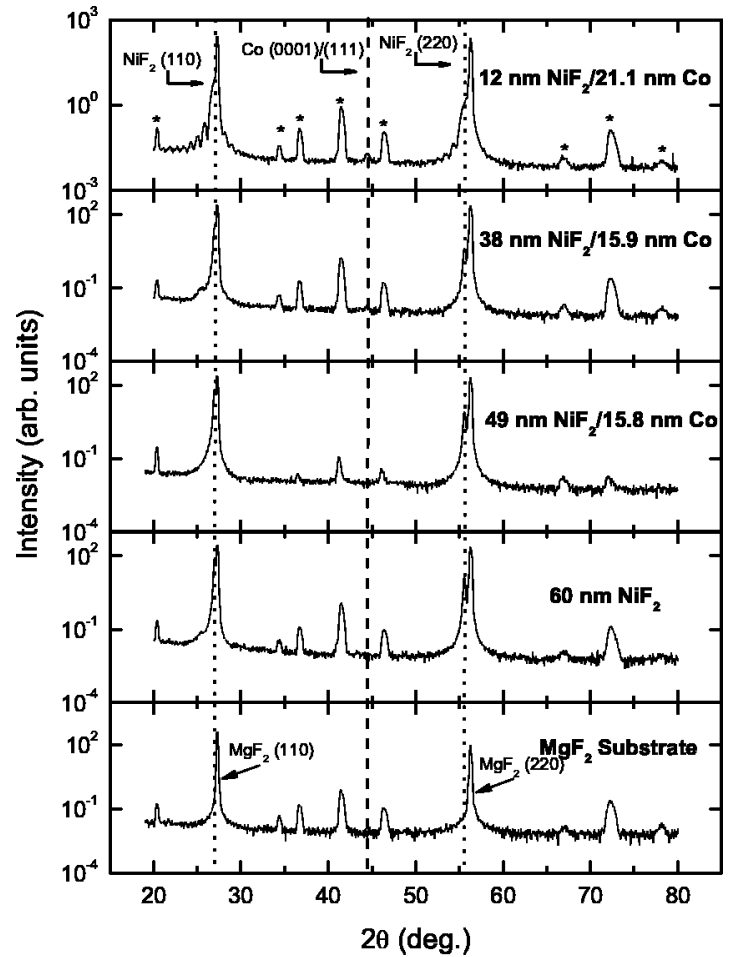

FIG. 3. $\theta-2 \theta$ x-ray diffraction scans of the samples used in this study. The observed $\mathrm{NiF}_{2}$ reflections are indicated by the dotted vertical lines. The dashed vertical line is the position of the Co fcc (111) or hcp (0001) reflection. The bottom panel is a scan of the substrate without overlayers, and the $\mathrm{MgF}_{2}$ (110) and (220) peaks are labeled. The other peaks in the bottom panel, labeled with a $(*)$ on the top panel, are unidentified substrate impurities.

On the other hand, the Co surface is much rougher than the $\mathrm{NiF}_{2}$ surface (see Table I), as is the case in $\mathrm{FeF}_{2} /$ Co bilayers.

Figure 3 shows the out-of-plane x-ray diffraction scan for the samples used in this study. The films are clearly strongly (110) oriented. For the two thinnest samples, finite-size fringes can be observed about the (110) and (220) reflections, indicating that the top and bottom surfaces of the $\mathrm{NiF}_{2}$ films are very smooth, in qualitative agreement with the reflectivity data. For the two thinnest samples, a Co hcp (0001) or fcc (111) peak is also observed. A Lorentzian rocking curve was observed for the $\mathrm{NiF}_{2}$ (220) reflection with a full width at half maximum (FWHM) of $0.19^{\circ}$, which is significantly smaller than widths of $\mathrm{FeF}_{2}$ films of similar thickness grown on $\mathrm{MgO}$ (100) (twinned with $\mathrm{FWHM}=1.8^{\circ}$ ) and on $\mathrm{MgF}_{2}$ (110) (single crystalline with FWHM $=0.50^{\circ}$ ). This is illustrated in Fig. 4.

TABLE I. Structural parameters obtained from the X-ray reflectivity shown in Fig. 2. $t$ are the thicknesses and $\sigma$ are the interface roughness parameters. All values in nanometers. Uncertainties for $t$ are $\approx \pm 0.2 \mathrm{~nm}$ and $\approx \pm 0.2 \mathrm{~nm}$ for $\sigma$.

\begin{tabular}{lcccccc}
\hline \hline $\mathrm{NiF}_{2} t$ & $\mathrm{Co} t$ & $\mathrm{MgF}_{2}$ & $\sigma \mathrm{NiF}_{2} /$ subst & $\sigma \mathrm{Co} / \mathrm{NiF}_{2}$ & $\sigma \mathrm{MgF}_{2} / \mathrm{Co}$ & $\sigma$ air/ $\mathrm{MgF}_{2}$ \\
\hline 12.0 & 21.1 & 5.0 & 0.3 & 0.2 & 1.9 & 1.0 \\
38.0 & 15.9 & 5.0 & 0.4 & 0.3 & 1.2 & 0.8 \\
49.0 & 15.8 & 5.0 & 0.3 & 0.3 & 1.0 & 1.0 \\
\hline \hline
\end{tabular}




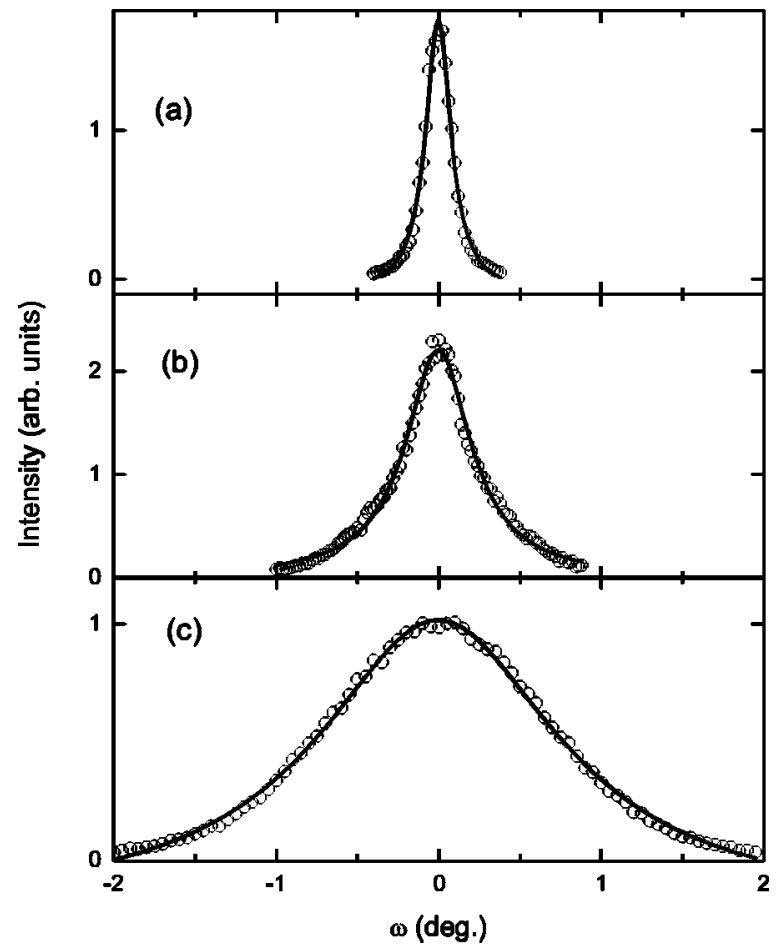

FIG. 4. Rocking curves for the (220) peaks of (a) $60 \mathrm{~nm} \mathrm{NiF} 2$ grown on $\mathrm{MgF}_{2}$ (110); (b) $68 \mathrm{~nm} \mathrm{FeF}_{2}$ grown on $\mathrm{MgF}_{2}$ (110); and (c) $69 \mathrm{~nm} \mathrm{FeF}_{2}$ grown on $\mathrm{MgO}$ (100). All samples were grown under similar conditions. Samples grown on $\mathrm{MgF}_{2}$ are epitaxial single crystalline while the sample grown on $\mathrm{MgO}(100)$ is twinned with two equivalent in-plane $c$ axes. The circles are the data and the solid curves are fits to Lorentzian line shapes. The widths of the fits are $0.19^{\circ}, 0.50^{\circ}$, and $1.8^{\circ}$, respectively.

X-ray diffraction $\phi$ scans demonstrated that the $\mathrm{NiF}_{2}$ grows epitaxially on the $\mathrm{MgF}_{2}$. Figures 5(a) and 5(b) show the in-plane $\phi$ scans of the $60 \mathrm{~nm}$ thick single $\mathrm{NiF}_{2}$ film and its substrate. The scans were carried out with the incident beam and the detector fixed at the $\mathrm{NiF}_{2}$ (332) and $\mathrm{MgF}_{2}$ (332) Bragg conditions while the sample was rotated about

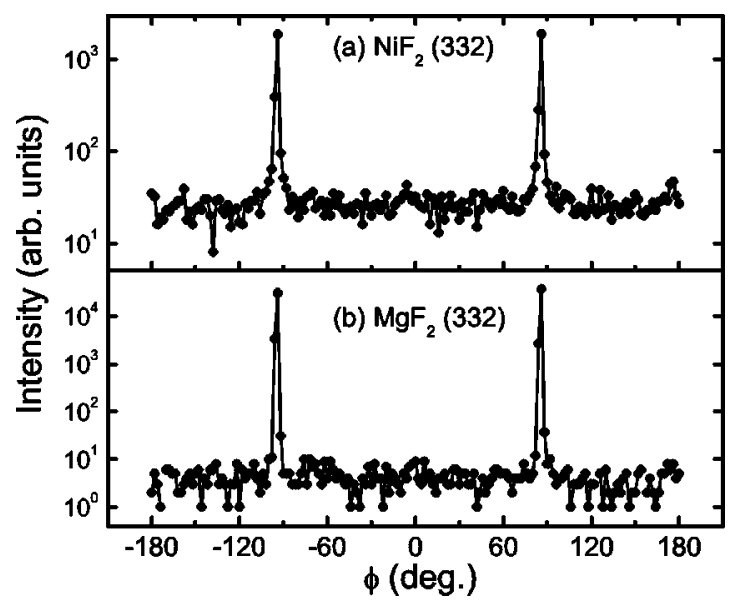

FIG. 5. Typical $\phi$ scans for the (a) $\mathrm{NiF}_{2}$ (332) and (b) $\mathrm{MgF}_{2}$ (332) Bragg reflections used to determine the epitaxial relationship of $\mathrm{NiF}_{2}$ to the substrate: $\mathrm{NiF}_{2}[001] \| \mathrm{MgF}_{2}[001]$ and $\mathrm{NiF}_{2}[110] \|$ $\mathrm{MgF}_{2}$ [110]. Lines are guides to the eye.

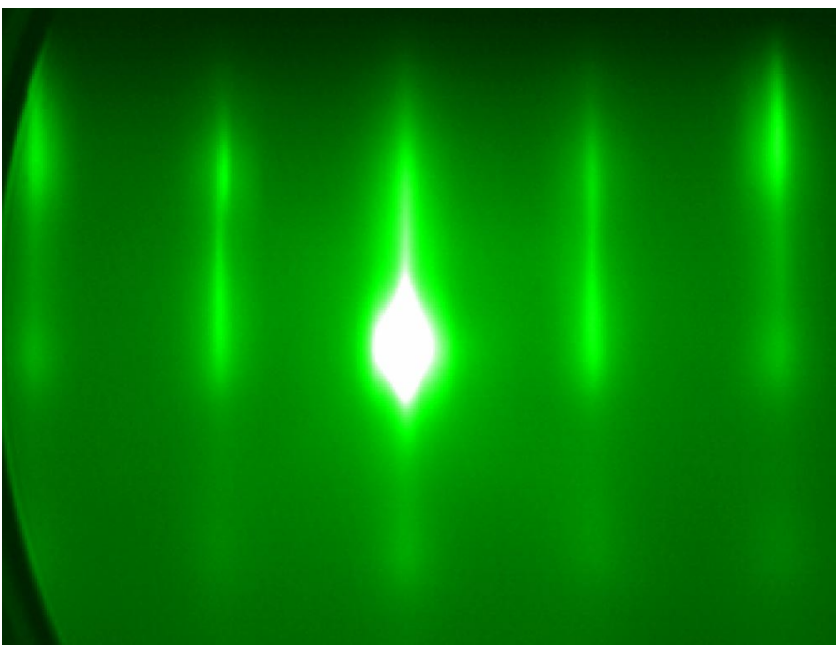

FIG. 6. RHEED pattern of the $60 \mathrm{~nm} \mathrm{NiF}$ sample with the electron beam incident parallel to $\mathrm{NiF}_{2}$ [110] direction.

the surface normal. Combining the out-of-plane and in-plane scans, the epitaxial relationship was determined to be $\mathrm{NiF}_{2}$ [001] $\| \mathrm{MgF}_{2}$ [001] and $\mathrm{NiF}_{2}$ [110] $\| \mathrm{MgF}_{2}$ [110]. This was confirmed by RHEED patterns obtained after the $\mathrm{NiF}_{2}$ layer growth, as shown in Fig. 6. This pattern, obtained with the incident beam along the $\mathrm{NiF}_{2}$ [110] direction for the $60 \mathrm{~nm}$ thick sample, was only twofold symmetric as a result of the twofold symmetry of the (110) surface. Furthermore, the streaky nature of the pattern qualitatively indicates that the surface is crystalline and smooth. The in-plane lattice parameters were determined from Bragg reflections with a component of $\vec{q}$ being parallel and perpendicular to the in-plane [001] direction. Figure 7 shows $\theta-2 \theta$ scans of the $\mathrm{NiF}_{2}$ (332) and (420) Bragg reflections, as well as the fittings to Gaussian line shapes. After transforming the base vectors from bct $a=[100], b=[010]$, and $c=[001]$ to $a^{\prime}=[110], b^{\prime}=[110]$, and

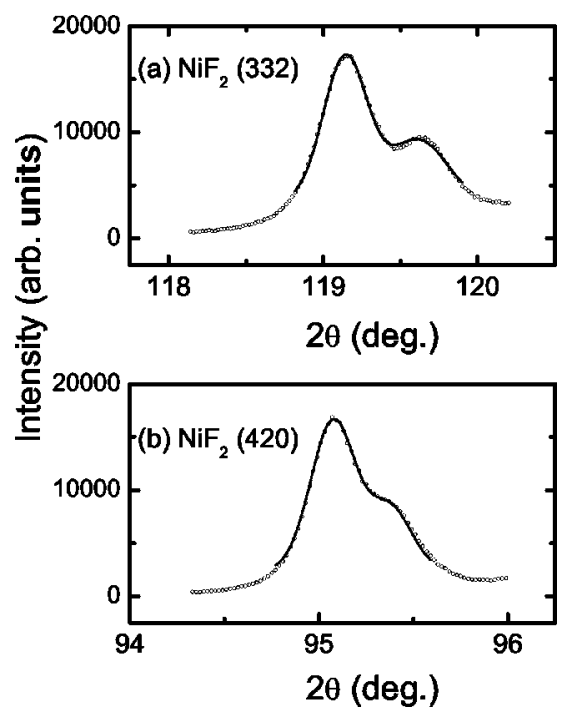

FIG. 7. $\theta-2 \theta$ scans of the (332) and (420) peaks of a $60 \mathrm{~nm} \mathrm{NiF}$ sample. The two peaks correspond to the $\mathrm{Cu} \mathrm{K}_{\alpha 1}$ and $\mathrm{Cu} \mathrm{K}_{\alpha 2}$ wavelengths. The data are the dots and the curves are fits to two Gaussians. 
TABLE II. Lattice constants $d_{h k l}$ for $\mathrm{NiF}_{2}$. Bulk $\mathrm{NiF}_{2}$ and $\mathrm{MgF}_{2}$ values obtained from Refs. 10 and 22, respectively. $\mathrm{NiF}_{2}$ thin-film values obtained from this work. The $60 \mathrm{~nm}$ sample does not have a Co overlayer. Values in nanometers. Uncertainties for thin-film lattice parameters are $\pm 0.0002 \mathrm{~nm}$.

\begin{tabular}{lccc}
\hline \hline Sample & $d_{110}$ & $d_{110}$ & $d_{001}$ \\
\hline Bulk MgF & 0.3267 & 0.3267 & 0.3040 \\
Bulk NiF$_{2}$ & 0.3289 & 0.3289 & 0.3084 \\
$60 \mathrm{~nm} \mathrm{NiF}_{2}$ & 0.3307 & 0.3272 & 0.3056 \\
$49 \mathrm{~nm} \mathrm{NiF}_{2}$ & 0.3304 & & 0.3058 \\
$38 \mathrm{~nm} \mathrm{NiF} 2$ & 0.3304 & & 0.3061 \\
$12 \mathrm{~nm} \mathrm{NiF}_{2}$ & 0.3302 & & 0.3061 \\
\hline \hline
\end{tabular}

$c^{\prime}=[001]$, we see that the (332) and (420) reflections in the bct coordinate system become (302) and (310) reflections in the new indexing system. Because the [110] direction is the growth direction, and the $a^{\prime}$ lattice parameter is known from the out-of-plane scan, $c^{\prime}$ and $b^{\prime}$ can be easily calculated. Results are shown in Table II, along with the lattice constant of bulk $\mathrm{NiF}_{2}$ and $\mathrm{MgF}_{2}$ and the [110] and [001] lattice parameters for the other samples used in this study. From the table it is clear that the $\mathrm{NiF}_{2}$ contracts along [001] and [110] directions due to the smaller lattice constant of the $\mathrm{MgF}_{2}$ substrate. However, along the surface normal, such restriction does not exist, leading to a lattice expansion along that direction. Although $d_{110}$ was not directly measured for the thinner films, it is safe to assume that it is similar to the $60 \mathrm{~nm}$ film value because of the similar $d_{001}$ values. Note that within the uncertainty of our measurements there is very little variation of the lattice parameters as a function of $\mathrm{NiF}_{2}$ thickness, indicating that the strain is similar in all of our thin-film samples. The width of diffraction peaks with $\vec{q}$ perpendicular to the surface and partially in the plane ${ }^{17}$ indicated that the out-of-plane and in-plane coherence lengths for the $60 \mathrm{~nm}$ film were $35.7 \mathrm{~nm}$ and $29.4 \mathrm{~nm}$, respectively.

\section{B. General magnetic properties of the samples}

Representative magnetization hysteresis loops for the $49 \mathrm{~nm}$ sample are shown in Fig. 8. Figures 9-11 show how the exchange bias, coercivity $\left(H_{C}\right)$, and neutron magnetic peak intensity depend on temperature for the $49 \mathrm{~nm}, 38 \mathrm{~nm}$, and $12 \mathrm{~nm}$ samples, respectively. In all the magnetization measurements $H_{C F}=2 \mathrm{kOe}$ was applied parallel to the $\mathrm{NiF}_{2}$ [110] direction. Note that for the two thicker samples $H_{C}$ has a peak that does not coincide with $T_{B}$, unlike what is observed in single crystal $\mathrm{FeF}_{2} / \mathrm{Co}$ and twinned $\mathrm{FeF}_{2} / \mathrm{Fe}$ samples, where the position of the peak coincides with $T_{B}{ }^{23-25}$ The origin of this peak in $\mathrm{FeF}_{2}$ has been attributed to short-range order above $T_{N},{ }^{25}$ to the properties of the surface antiferromagnetic susceptibility, ${ }^{23}$ or to uncompensated spins due to domain formation in the antiferromagnet. ${ }^{26} \mathrm{~A}$ similar mechanism may be responsible for the behavior in $\mathrm{NiF}_{2}$. Also note that for the $49 \mathrm{~nm}$ sample $H_{E}>0$, which also occurs for $\mathrm{FeF}_{2}$ cooled in high fields. ${ }^{27}$ We have verified that $H_{E}<0$ at $T=5 \mathrm{~K}$ when cooling in $H_{C F}=1 \mathrm{kOe}$. These issues

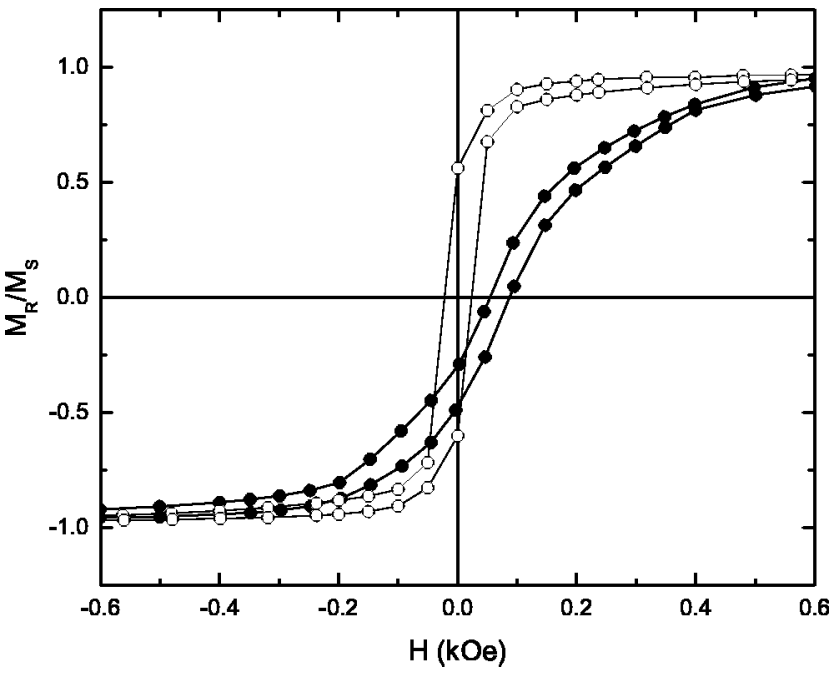

FIG. 8. Magnetization hysteresis loops for the $49 \mathrm{~nm}$ $\mathrm{NiF}_{2} / 15.8 \mathrm{~nm}$ Co bilayer sample. Data obtained at $T=90 \mathrm{~K}(\bigcirc)$ and $T=5 \mathrm{~K}(\bullet)$, the latter after field cooling in $H_{C F}=2 \mathrm{kOe} . H$ and $H_{C F}$ were applied along the $\mathrm{NiF}_{2}$ [110] direction.

will be studied in more detail in a future publication.

\section{Enhancement of the $\mathrm{NiF}_{2}$ Néel temperature}

Table III summarizes $T_{N}$ and $T_{B}$ for the different samples. Except for the thinnest sample, $T_{B}, T_{N} \approx 81 \mathrm{~K}$, a significant

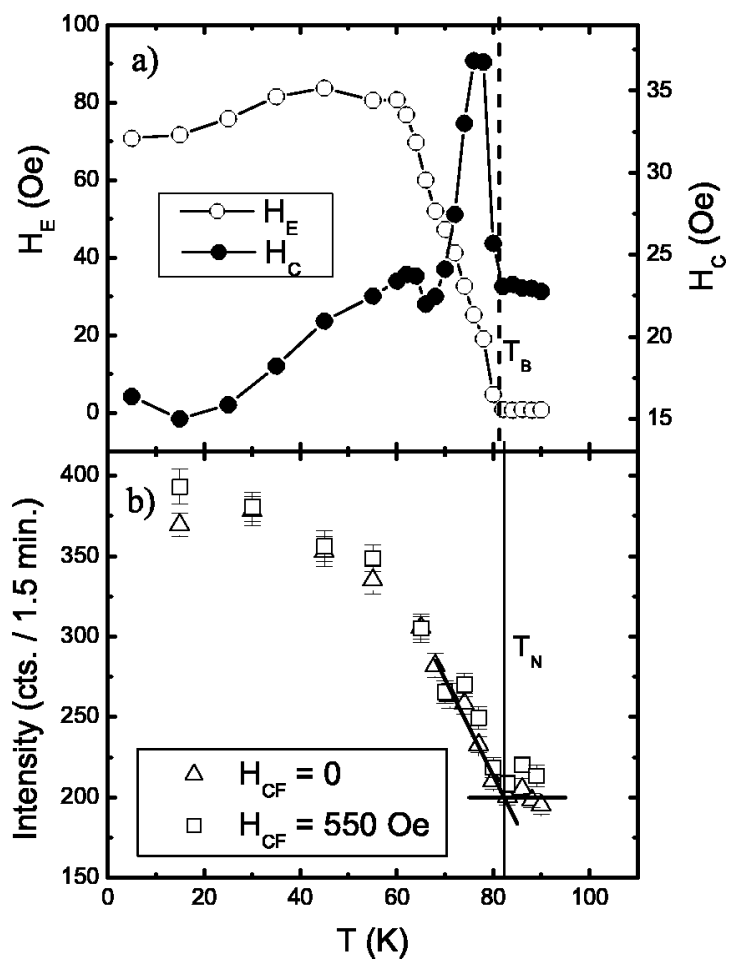

FIG. 9. (a) Exchange bias and coercive field of a $49 \mathrm{~nm}$ $\mathrm{NiF}_{2} / 15.8 \mathrm{~nm}$ Co bilayer as a function of temperature after cooling in $H_{C F}=2$ kOe. (b) Temperature dependence of the $(001) \mathrm{NiF}_{2}$ neutron magnetic peak intensity after field cooling in $H_{C F}=550$ Oe and $H_{C F}=0$. Solid lines are linear fits to the data points close to $T_{N}$ for $H_{C F}=0$. The intersection of the lines yields $T_{N}$. The dashed vertical line indicates $T_{B}$ and the solid vertical line indicates $T_{N}$. 


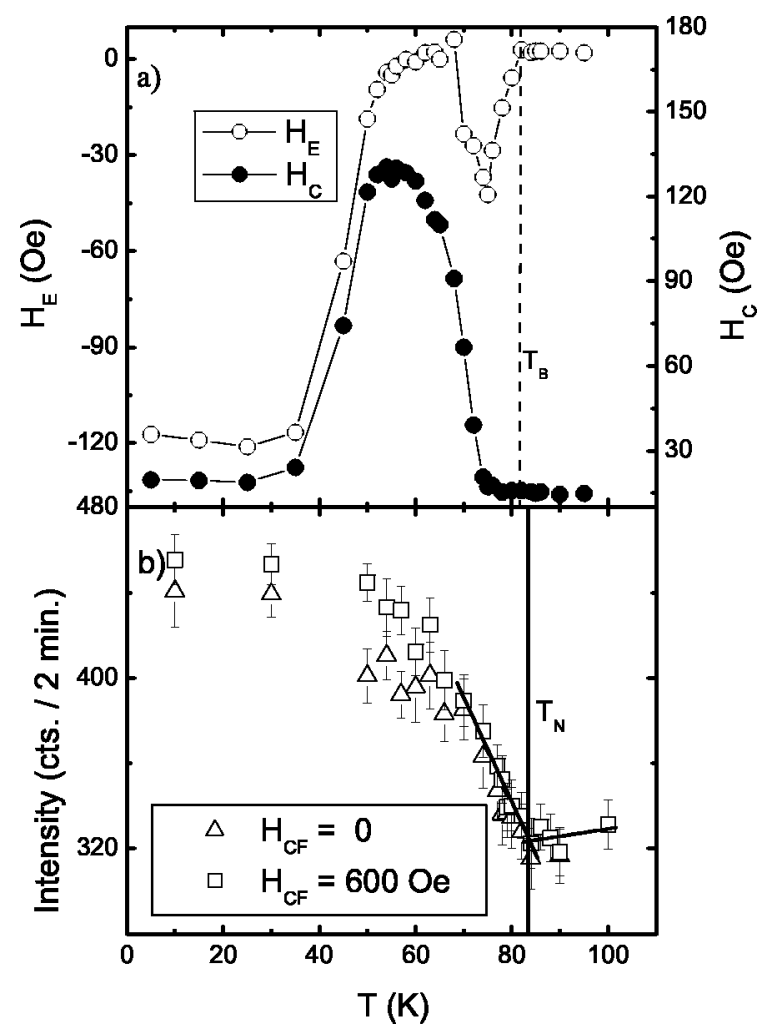

FIG. 10. (a) Exchange bias and coercive field of a $38 \mathrm{~nm}$ $\mathrm{NiF}_{2} / 15.9 \mathrm{~nm}$ Co bilayer as a function of temperature after cooling in $H_{C F}=2 \mathrm{kOe}$. (b) Temperature dependence of the (001) $\mathrm{NiF}_{2}$ neutron magnetic peak intensity after field cooling in $H_{C F}=600 \mathrm{Oe}$ and $H_{C F}=0$. Solid lines are linear fits to the data points close to $T_{N}$ for $H_{C F}=0$. The intersection of the lines yields $T_{N}$. The dashed vertical line indicates $T_{B}$ and the solid vertical line indicates $T_{N}$.

enhancement over the accepted $T_{N}$ of bulk $\mathrm{NiF}_{2}(73.2 \mathrm{~K})$. The value for the thinnest sample, obtained from Fig. 11, is actually a lower, conservative bound. The actual Néel temperature for this sample could be as high as $85 \mathrm{~K}$, but the relatively weak signal near $T_{N}$ makes it impossible to obtain a better measurement. An important question is whether $T_{B}$ is enhanced by a larger $T_{N}$ of the $\mathrm{NiF}_{2}$ film. If so, the question is whether it results from a proximity effect, where the Co film, due to its high Curie temperature $\left(T_{C}=1388 \mathrm{~K}\right.$ in bulk form), causes the $\mathrm{NiF}_{2}$ to order at an unusually high temperature. As mentioned above, this has been observed experimentally in $\mathrm{Fe}_{3} \mathrm{O}_{4} / \mathrm{CoO}$ multilayers and has also been confirmed theoretically using Monte Carlo simulations. ${ }^{28}$ Note that the values of $T_{N}$ obtained from neutron data with and without field cooling for the $49 \mathrm{~nm}$ and $38 \mathrm{~nm}$ samples are almost identical, taking into account the error bars, indicating that $T_{N}$ does not depend on $H_{C F}$. The background of the magnetic (001) peak is due to the nuclear contribution of the $\mathrm{MgF}_{2}$ substrate. The integrated intensity of (001) peak (not shown here) gives exactly the same temperature dependence as that of the peak intensity.

We also measured the magnetization along the [110] direction of a $60 \mathrm{~nm}$ thick single $\mathrm{NiF}_{2}$ film with no Co overlayer, in addition to neutron-diffraction measurements. Figure 12(a) shows the $\mathrm{NiF}_{2}(001)$ peak intensity as a function

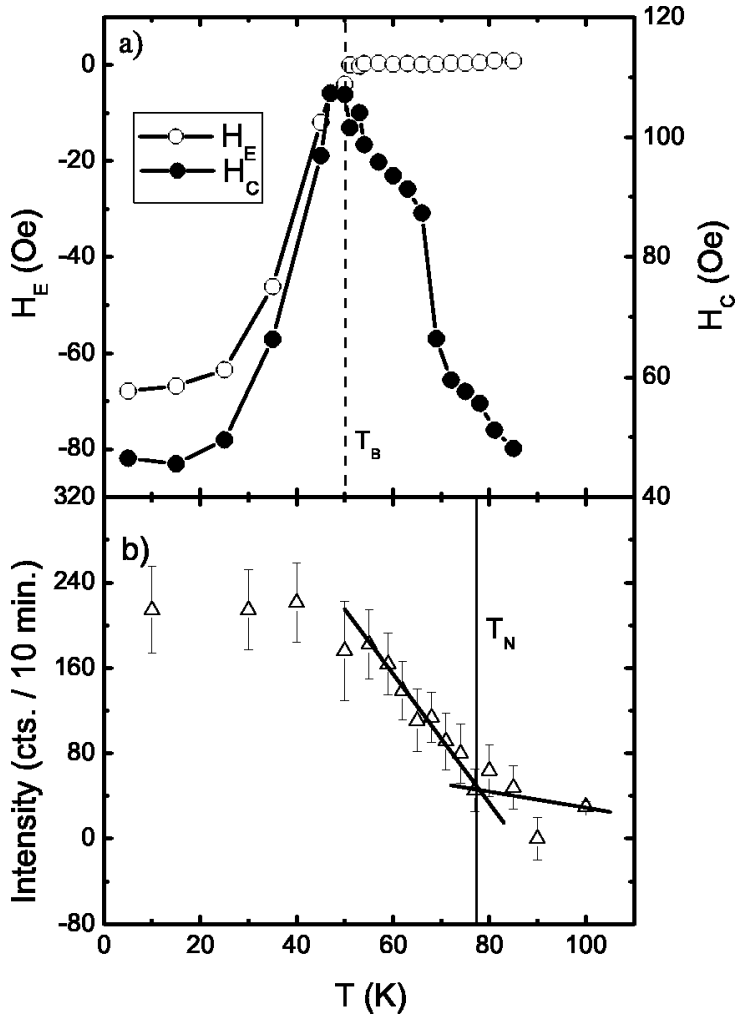

FIG. 11. (a) Exchange bias and coercive field of a $12 \mathrm{~nm}$ $\mathrm{NiF}_{2} / 21.1 \mathrm{~nm}$ Co bilayer as a function of temperature after cooling in $H_{C F}=2 \mathrm{kOe}$. (b) Temperature dependence of the (001) $\mathrm{NiF}_{2}$ neutron magnetic integrated peak intensity after field cooling in $H_{C F}$ $=0$. Solid lines are linear fits to the data points close to $T_{N}$ for $H_{C F}=0$. The intersection of the lines yields $T_{N}$. The dashed vertical line indicates $T_{B}$ and the solid vertical line indicates $T_{N}$.

of temperature after field cooling $\left(H_{C F}=550 \mathrm{Oe}\right)$ and zerofield cooling. Note that $T_{N}=81 \mathrm{~K}$, as was the case for the bilayer samples shown in Figs. 9 and 10, indicating that the enhancement of $T_{N}$ is not due to the proximity effect. Figure 12(b) shows magnetic susceptibility $\chi$, defined as $\chi=M / H$, of this sample with a $2.3 \mathrm{kOe}$ field applied along the $\mathrm{NiF}_{2}$ [110] direction as a function of temperature, after cooling from $T=100 \mathrm{~K}$ to $T=5 \mathrm{~K}$ in $H_{C F}=2 \mathrm{kOe}$. Because $\mathrm{NiF}_{2}$ is only a weak ferromagnet, the peak in $\chi$ corresponds to $T_{N} \cdot{ }^{14}$ The inset of Fig. 12(b) shows $d \chi / d T$, from which we determined that the peak of $\chi$ is at $T_{N}=79.7 \mathrm{~K}$. This result indeed agrees very well with the neutron-scattering result; the small disagreement may be due to different thermometry set-ups.

TABLE III. Néel $\left(T_{N}\right)$ and blocking $\left(T_{B}\right)$ temperatures for different samples. $T_{N}$ was determined from neutron diffraction and $T_{B}$ from magnetization measurements. The $60 \mathrm{~nm}$ sample has no Co overlayer and therefore $T_{B}$ is not available.

\begin{tabular}{ccc}
\hline \hline $\mathrm{NiF}_{2}$ thickness $(\mathrm{nm})$ & $T_{B}(\mathrm{~K})$ & $T_{N}(\mathrm{~K})$ \\
\hline 12 & $53.1 \pm 0.5$ & $78(-1+7)$ \\
38 & $81.5 \pm 0.5$ & $83.7 \pm 2$ \\
49 & $81.0 \pm 0.5$ & $82.3 \pm 2$ \\
60 & & $81.4 \pm 2$ \\
\hline \hline
\end{tabular}



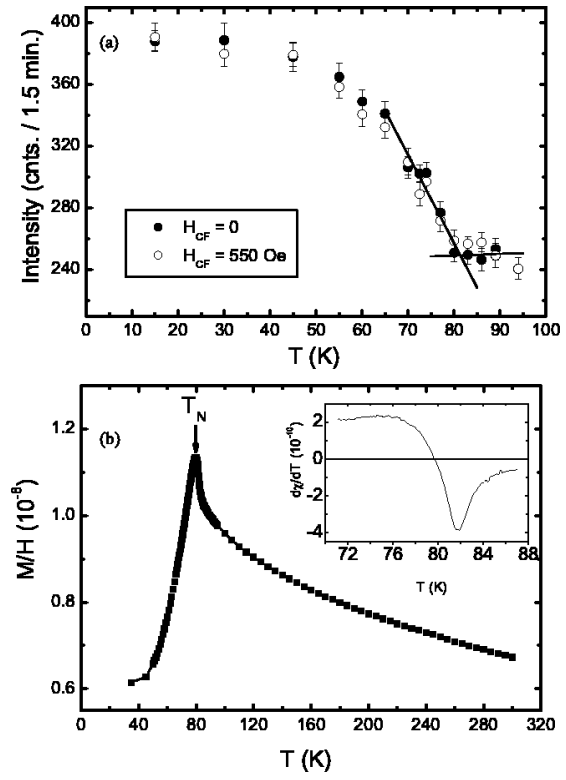

FIG. 12. (a) Temperature-dependent intensity of the (001) magnetic reflection for the $60 \mathrm{~nm} \mathrm{NiF}_{2}$ film after field cooling and zerofield cooling. Note that $T_{N}=81 \mathrm{~K}$. Lines are linear fits to the data close to $T_{N}$ for $H_{C F}=0$. (b) Magnetic susceptibility $\chi=M / H$ as a function of temperature after cooling the sample from $T=100 \mathrm{~K}$ to $T=5 \mathrm{~K}$ in $H_{C F}=2 \mathrm{kOe}$. Both the cooling field and the measuring field $(H=2300 \mathrm{Oe})$ are parallel to the $\mathrm{NiF}_{2}$ [110] direction. The inset shows the first derivative of $\chi$ with respect to the temperature. $T_{N}=79.7 \mathrm{~K}$ from the point at which $d \chi / d T=0$.

The values of $T_{N}$ and $T_{B}$ for all the samples are shown in Table III.

To interpret the enhancement of the films' $T_{N}$ with respect to bulk material, we first note that bulk $\mathrm{NiF}_{2}$ has lattice constants that are similar to those of the $\mathrm{MgF}_{2}$ substrate. As indicated by the $\mathrm{x}$-ray structural analysis above (Table II), the $\mathrm{NiF}_{2}$ films grow strained in order to be lattice matched to the substrate crystal structure. This strain causes a significant orthorhombic distortion of the lattice throughout the entire thickness. It is therefore important to determine whether this distortion is responsible for the enhancement of $T_{N}$. Unfortunately, the dependence of $T_{N}$ on pressure or lattice parameters has not been studied in $\mathrm{NiF}_{2}$, so it is not possible to directly compare our results with experimental data. One may assess this issue by using results from other magnetic materials. Specifically, in many antiferromagnets a change in the unit-cell volume is related to a change in effective exchange interaction by ${ }^{29}$

$$
\frac{d(\ln J)}{d(\ln V)}=-\gamma_{m},
$$

where $V$ is the volume of the unit cell, $d(\ln J) \approx \ln \left(J_{s} / J_{\mathrm{o}}\right)$, and $d(\ln V) \approx \ln \left(V_{s} / V_{\mathrm{o}}\right)$. Here $J_{s}$ and $V_{s}$ refer to the sample exchange and volume, respectively, and $J_{\mathrm{o}}$ and $V_{\mathrm{o}}$ refer to the exchange and volume of the bulk crystal. This $\gamma_{m}$ is an effective magnetic Grüneisen constant which for a wide array of magnetic materials $\gamma_{m}=10 / 3$, including $\mathrm{FeF}_{2}$ (Ref. 30) and $\mathrm{MnF}_{2} \cdot{ }^{31}$ Assuming that $\mathrm{NiF}_{2}$ obeys the 10/3 law; using

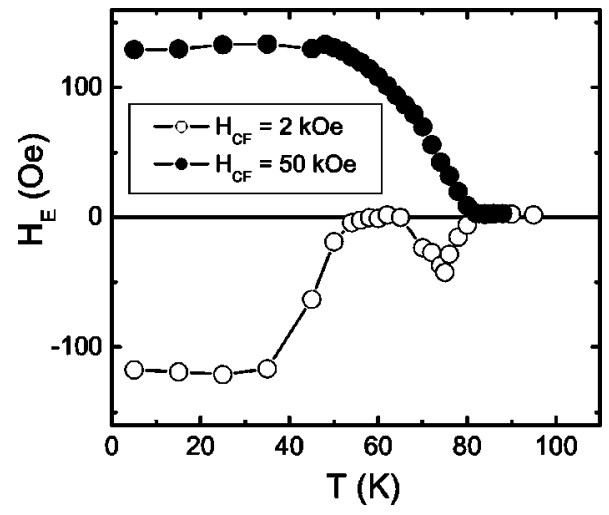

FIG. 13. Exchange bias for two cooling fields as a function of temperature for the $38 \mathrm{~nm} \mathrm{NiF}_{2} / 15.9 \mathrm{~nm}$ Co sample.

the mean-field result that $T_{N} \propto J$ (known to be accurate for calculating changes in $T_{N}$ ) (Ref. 32); and using $V$ $=2 d_{110} d_{110} d_{001}$, we obtain that $\Delta T_{N}=2.4 \mathrm{~K}$. This is smaller than our experimental value of $\approx 8 \mathrm{~K}$ by a factor of 3 . The lattice mismatch at low temperatures between $\mathrm{MgF}_{2}$ and $\mathrm{NiF}_{2}$ is smaller than at room temperature, ${ }^{33}$ so the expected change in $T_{N}$ discussed here is an upper bound. The large relative change of the $c$-axis lattice parameter would seem to indicate that a change in $J_{1}$ might be responsible for the enhanced $T_{N}$, but this is unlikely because $J_{1}$ is more than 60 times smaller in magnitude than $J_{2}$.

Our results therefore suggest that $\gamma_{m}$ is anomalous for $\mathrm{NiF}_{2}$. One possibility is that the fluorine ions are displaced from their equilibrium positions, independent of the lattice expansion or contraction, which could have a significant effect on the exchange interactions. $\mathrm{NiF}_{2}$ is known to have a significant magnetostrictive shift of the fluorine ions at low $T$ with respect to the value above $T_{N}$, unlike $\mathrm{FeF}_{2} \cdot{ }^{33,34}$ Further neutron and $\mathrm{x}$-ray measurements are needed todetermine whether this is the case.

\section{Reentrant exchange bias}

Figure 10 shows the exchange bias and the $\mathrm{NiF}_{2}(001)$ peak intensity as a function of temperature in the $38 \mathrm{~nm}$ bilayer sample. For a small cooling field $\left(H_{C F}=2 \mathrm{kOe}\right) H_{E}$ shows a reentrant behavior. In other words, it goes to zero at $T \sim 55 \mathrm{~K}$ and then becomes negative before it vanishes again at $T=81 \mathrm{~K}$. On the other hand, if the cooling field is sufficiently large $\left(H_{C F}=50 \mathrm{kOe}\right.$, see Fig. 13$), H_{E}$ remains positive before it vanishes at the same blocking temperature, $T_{B}=81 \mathrm{~K}$. This behavior is similar to the change in sign of $H_{E}$ as a function of $T$ in $\mathrm{Fe}_{x} \mathrm{Zn}_{1-x} \mathrm{~F}_{2} / \mathrm{Co}$ bilayers, with $x$ $\sim 0.80$, where $H_{E}$ can be zero at an intermediate temperature for moderate cooling fields. ${ }^{35}$ For the case of the $\mathrm{NiF}_{2}$, when $H_{E}$ goes to zero as the sample is warmed, instead of becoming positive at higher temperatures, it becomes negative once again. It is unclear whether the change in sign of $H_{E}$ in $\mathrm{Fe}_{x} \mathrm{Zn}_{1-x} \mathrm{~F}_{2}$ and the reentrant effect in $\mathrm{NiF}_{2}$ are related. However, it is possible that for $\mathrm{NiF}_{2}$ this is a result of a reorientation of the antiferromagnetic domains from the (100) plane to the (010) plane or vice versa. A similar reentrant effect, although less marked, has been observed in $\mathrm{FePt}_{3} / \mathrm{Fe}$ 
bilayers $^{36}$ and its origin is believed to result from a spin reorientation in the antiferromagnet, similar to the one discussed here, that has been observed via neutron scattering. ${ }^{37}$ Further neutron-diffraction measurements of the magnetic (100) peak are planned to determine whether this is true. Unfortunately a substrate contamination peak precluded us from performing these measurements with high accuracy in these samples, so better substrates will be needed.

\section{E. Reduction of $T_{B}$ in thin $\mathrm{NiF}_{2}$}

Figure 11(a) shows $H_{E}$ as a function of temperature for a $12 \mathrm{~nm}$ thick $\mathrm{NiF}_{2}$ sample. In this case $T_{B}$ is reduced to $T$ $\approx 53 \mathrm{~K}$. However, the integrated $\mathrm{NiF}_{2}(001)$ peak intensity, as shown in Fig. 11(b), indicates that $T_{N} \approx 78 \mathrm{~K}$, with an uncertainty of approximately $-1+7 \mathrm{~K}$. Hence, $T_{N}$ is certainly above $70 \mathrm{~K}$, and is possible as high as $85 \mathrm{~K}$. Note that all the data in Fig. 11(b) are plotted after subtracting the nuclear $\mathrm{MgF}_{2}(001)$ peak measured at $T=90 \mathrm{~K}$. This shows that the reduced $T_{B}$ is not due to a diminished $T_{N}$, but is probably due to the AF domains in the $\mathrm{NiF}_{2}$ becoming unpinned above $T$ $=53 \mathrm{~K}$. This is consistent with measurements in single crystals that suggest that the magnetization in the $a-b$ plane becomes isotropic close to $T_{N}{ }^{14}$ It is not surprising that for small $\mathrm{NiF}_{2}$ thicknesses this effect would be magnified because the total magnetic anisotropy energy is proportional to the volume, and therefore, the thickness of the film. It is also interesting to note that the temperature at which $H_{E}$ goes to zero for the first time $(53 \mathrm{~K})$ in the $38 \mathrm{~nm}$ sample coincides with $T_{B}$ for the $12 \mathrm{~nm}$ sample. Further measurements are required to determine whether this is a coincidence, or if $T$ $=50 \mathrm{~K}$ is the temperature where the magnetic in-plane anisotropy of the $\mathrm{NiF}_{2}$ "softens" up. In any case, this is a clear experimental demonstration that, in general, a low $T_{B}$ is not necessarily due to a lower $T_{N}$, due to finite-size effects, for example, but could be due to other factors, such as a smaller effective anisotropy energy that can no longer withstand its magnetic structure at higher temperatures.

\section{CONCLUSIONS}

In summary, (110) $\mathrm{NiF}_{2}$ was epitaxially grown on (110) $\mathrm{MgF}_{2}$ substrates via MBE. In thicker $\mathrm{NiF}_{2}, T_{N}$ and $T_{B}$ are significantly larger than the bulk value. This enhancement of $T_{N}$ is likely to be a result of the strain in $\mathrm{NiF}_{2}$ due to a small lattice mismatch between the $\mathrm{NiF}_{2}$ films and the $\mathrm{MgF}_{2}$ substrates. In order to calculate the enhancement of $T_{N}$ due to the strain in $\mathrm{NiF}_{2}$, the dependence of $J_{2}$ not only on the lattice parameters, but possibly also on the position of the fluorine ions is needed. A reentrant exchange bias behavior was also observed for the $38 \mathrm{~nm}$ sample. For the thinnest, $12 \mathrm{~nm}$ sample, $T_{B} \ll T_{N}$, indicating that the antiferromagnet's anisotropy is not enough to maintain the exchange bias at higher temperatures below $T_{N}$, even though long-range order in $\mathrm{NiF}_{2}$ is maintained.

\section{ACKNOWLEDGMENTS}

This work was supported in part by the National Science Foundation (Grant No. EPS-0083046 ) at WVU.
*Present address: Department of Physics, University of California, Irvine, CA 92697-4560.

${ }^{1}$ W. H. Meiklejohn and C. P. Bean, Phys. Rev. 102, 1413 (1956).

${ }^{2}$ J. Nogués and I. K. Schuller, J. Magn. Magn. Mater. 192, 203 (1999).

${ }^{3}$ A. E. Berkowitz and K. Takano, J. Magn. Magn. Mater. 200, 552 (1999).

${ }^{4}$ S. Soeya, S. Nakamura, T. Imgawa, and S. Narishige, J. Appl. Phys. 77, 5838 (1995).

${ }^{5}$ M. S. Lund, W. A. A. Macedo, K. Liu, J. Nogués, I. K. Schuller, and C. Leighton, Phys. Rev. B 66, 054422 (2002).

${ }^{6}$ S. S. P. Parkin and V. S. Speriosu, in Magnetic Properties of Low-Dimensional Systems II. New Developments, Proceedings of the Second Workshop, edited by L. M. Falicov, F. Mejía Lira, and J. L. Morán-López (Springer-Verlag, Berlin, 1990), p. 110.

${ }^{7}$ D. Lederman, C. A. Ramos, V. Jaccarino, and J. L. Cardy, Phys. Rev. B 48, 8365 (1993).

${ }^{8}$ P. J. van der Zaag, Y. Ijiri, J. A. Borchers, L. F. Feiner, R. M. Wolf, J. M. Gaines, R. W. Erwin, and M. A. Verheijen, Phys. Rev. Lett. 84, 6102 (2000).

${ }^{9}$ H. Yamazaki and J. Satooka, J. Magn. Magn. Mater. 240, 442 (2002).

${ }^{10}$ J. W. Stout and S. A. Reed, J. Am. Chem. Soc. 76, 5279 (1954).

${ }^{11}$ T. Moriya, Phys. Rev. 117, 635 (1960).

${ }^{12}$ M. T. Hutchings, M. F. Thorpe, R. J. Birgeneau, P. A. Fleury, and
H. J. Guggenheim, Phys. Rev. B 2, 1362 (1970).

${ }^{13}$ J. W. Stout and E. Catalano, J. Chem. Phys. 23, 1284 (1955).

${ }^{14}$ A. S. Borovik-Romanov, A. N. Bazhan, and N. M. Kreines, Zh.

Eksp. Teor. Fiz. 64, 1367 (1973) [Sov. Phys. JETP 37, 695 (1973)]

${ }^{15}$ A. S. Pavlovic, Phys. Rev. B 21, 3652 (1980).

${ }^{16}$ J. Nogués, T. J. Moran, D. Lederman, I. K. Schuller, and K. V. Rao, Phys. Rev. B 59, 6984 (1999).

${ }^{17}$ J. McChesney, M. Hetzer, H. Shi, T. Charlton, and D. Lederman, J. Mater. Res. 16, 1769 (2001).

${ }^{18}$ B. Vidal and P. Vincent, Appl. Opt. 23, 1794 (1984).

${ }^{19}$ T. C. Schulthess and W. H. Butler, Phys. Rev. Lett. 81, 4516 (1998).

${ }^{20}$ R. A. Erickson, Phys. Rev. 90, 779 (1953).

${ }^{21}$ H. Shi and D. Lederman, Phys. Rev. B 66, 094426 (2002).

${ }^{22}$ W. Baur, Acta Crystallogr., Sect. B: Struct. Crystallogr. Cryst. Chem. B32, 2200 (1976).

${ }^{23}$ C. Leighton, H. Suhl, M. J. Pechan, R. Compton, J. Nogués, and I. K. Schuller, J. Appl. Phys. 92, 1483 (2002).

${ }^{24}$ H. Shi, D. Lederman, and E. E. Fullerton, J. Appl. Phys. 91, 7763 (2002).

${ }^{25}$ M. Grimsditch, A. Hoffmann, P. Vavassori, H. Shi, and D. Lederman, Phys. Rev. Lett. 90, 257201 (2003).

${ }^{26}$ I. N. Krivorotov, C. Leighton, J. Nogués, I. K. Schuller, and E. D. Dahlberg, Phys. Rev. B 68, 054430 (2003). 
${ }^{27}$ J. Nogués, D. Lederman, T. J. Moran, and I. K. Schuller, Phys. Rev. Lett. 76, 4624 (1996).

${ }^{28}$ S. H. Tsai, D. P. Landau, and T. C. Schulthess, J. Appl. Phys. 93, 8612 (2003).

${ }^{29}$ D. Bloch, J. Phys. Chem. Solids 27, 881 (1966).

${ }^{30}$ G. A. Garcia and R. Ingalls, J. Phys. Chem. Solids 37, 211 (1975).

${ }^{31}$ K. C. Johnson and A. J. Sievers, Phys. Rev. B 10, 1027 (1974).

${ }^{32}$ G. K. Wertheim, H. J. Guggenheim, M. Butler, and V. Jaccarino, Phys. Rev. 178, 804 (1969).
${ }^{33}$ W. Jauch, Phys. Rev. B 44, 6864 (1991).

${ }^{34}$ A. Palmer and W. Jauch, Phys. Rev. B 48, 10304 (1993).

${ }^{35}$ H. Shi, D. Lederman, N. R. Dilley, R. C. Black, J. Diedrichs, K. Jensen, and M. B. Simmonds, J. Appl. Phys. 93, 8600 (2003).

${ }^{36}$ R. L. Compton, M. J. Pechan, S. Maat, and E. E. Fullerton, Phys. Rev. B 66, 054411 (2002).

${ }^{37}$ S. Maat, O. Hellwig, G. Zeltzer, E. E. Fullerton, G. J. Mankey, M. L. Crow, and J. L. Robertson, Phys. Rev. B 63, 134426 (2001). 